\title{
O sevofluorano em cadelas gestantes
}

\author{
The sevoflurane in pregnant bitches
}

\section{Lídia Mitsuko Matsubara $^{1}$ Valéria Nobre Leal de Souza Oliva ${ }^{2}$ Daniela Tozadore Gabas ${ }^{1}$ Lilian Bevilacqua $^{1}$ Sílvia Helena Venturolli Perri ${ }^{3}$}

\section{RESUMO}

Com este experimento, objetivou-se avaliar como a anestesia geral inalatória, com o sevofluorano, interfere nos parâmetros fisiológicos de cadelas gestantes. Nove cadelas sem raça definida, adultas, com idade média de três anos, foram submetidas ao mesmo procedimento anestésico em dois períodos distintos, sendo o primeiro na condição não gestante e o segundo aos 45 dias de gestação. Todas receberam acepromazina $\left(0,05 \mathrm{mg} \mathrm{kg}^{-1}\right)$ pela via intravenosa como medicação pré-anestésica, propofol $\left(5 \mathrm{mg} \mathrm{kg}^{-1}\right)$ pela mesma via e sevofluorano diluído em oxigênio. As variáveis estudadas foram freqüencia cardíaca e respiratória, pressão arterial sistólica, média e diastólica, temperatura retal, pressão parcial de $\mathrm{CO}_{2}$ ao final da expiração, saturação de oxigênio nas hemoglobinas, $p H$ sangüíneo, pressão parcial arterial de oxigênio, pressão parcial arterial de $\mathrm{CO}_{2}$, bicarbonato e excesso de base. Os parâmetros foram avaliados antes da medicação pré-anestésica (M0) e 15 minutos após (M1), 15 minutos após a estabilização da anestesia inalatória (M2) e, depois, a cada 15 minutos durante 60 minutos (M3, M4, M5 e M6), com exceção das variáveis hemogasométricas que foram avaliadas em M0, M2 e M6. A análise estatística foi realizada com Análise de Variância e teste de Tukey, sendo considerado o nível de significância de 5\%. A freqüencia cardíaca elevou-se na paciente gestante no momento basal e 15 minutos após a medicação pré-anestésica, sendo observado ainda diminuição da pressão arterial e da temperatura retal nas gestantes. Os resultados obtidos permitiram concluir que o protocolo anestésico não alterou as variáveis mensuradas neste experimento, podendo ser utilizado com segurança para as cadelas, se houver a necessidade de intervenção cirúrgica e/ou anestésica aos 45 dias de sua gestação.
Palavras-chave: anestesia inalatória, sevofluorano, cadelas, gestação.

\section{ABSTRACT}

The aim of this work was to evaluate how inhalation anesthesia with sevoflurane alters physiological parameters of pregnant bitches. Nine mixed breed bitches with mean age of 3 year-old were submitted to the same anesthetic procedure in two different moments: the first one envolving non-pregnant bitches and the second one 45 day pregnant bitches. All bitches received acepromazine $\left(0.05 \mathrm{mg} \mathrm{kg}^{-1}\right)$ intravenously as premedication, propofol $\left(5 \mathrm{mg} \mathrm{kg}^{-1}\right)$ by the same way and sevoflurane diluted in oxygen. The variables measured were: heart rate and respiratory rate, systolic, mean and diastolic blood pressure, rectal temperature, end-tidal carbon dioxide pressure, oxyhemoglobin saturation, $\mathrm{pH}$, oxygen tension, carbon dioxide tension, bicarbonate and base excess. The parameters were evaluated before (M0) and 15 minutes after tranquilization (M1), 15 minutes after parameters stabilization of inhalation anesthesia (M2) and every 15 minutes during 60 minutes (M3, M4, M5, M6), except $p H$ and blood gas values which were measured before tranquilization (M0), 15 minutes after parameters stabilization (M2) and at 60 minutes of anesthesia (M6). The heart rate increased in the pregnant bitch before and 15 minutes after tranquilization and there was blood pressure and rectal temperature decrease. The statistical evaluation used were Analysis of Variance and Tukey's test $(P<0.05)$. These results allowed the conclusion that the anesthetic protocol didn't change the variables measured, which can be employed safely in case of surgical and/or anesthetic procedure in 45 day pregnant bitches.

Key words: inhalation anesthesia, sevoflurane, bitches, pregnancy.

${ }^{1}$ Curso de Doutorado, Departamento de Anestesiologia Experimental, Faculdade de Medicina da Universidade Estadual Paulista (UNESP), Campus de Botucatu, SP, Brasil. Rua: Clóvis Pestana, 793 Jd. Dona Amélia, DCCRA, 16050-680, Araçatuba, SP, Brasil. Telefone (18) 3636-3200, ramal 3690. E-mail: matsubarara@fmvz.unesp.br. Autor para correspondência.

${ }^{2}$ Departamento de Clínica, Cirurgia e Reprodução Animal do Curso de Medicina Veterinária da UNESP, Campus de Araçatuba, SP, Brasil.

${ }^{3}$ Departamento de Apoio, Produção e Saúde Animal do Curso de Medicina Veterinária da UNESP, Campus de Araçatuba, SP, Brasil. 


\section{INTRODUÇÃO}

A anestesia em pacientes gestantes é um desafio para o anestesista, que deve procurar uma depressão mínima da mãe e dos fetos. A literatura disponível refere-se a procedimentos anestésicos em cesarianas (GREENE, 1995; THURMON et al., 1996), devido ao fato de essa condição específica ser a indicação mais comum para a utilização de anestesia geral durante a gestação.

A escolha do protocolo anestésico é baseada na segurança da mãe e do feto, conforto da paciente, conveniência do cirurgião e familiaridade com a técnica anestésica (THURMON et al., 1996), e a melhor técnica é aquela que causa pouca depressão fetal sem expor a mãe a riscos desnecessários (SCHNIDER \& LEVINSON, 1987).

Todos os anestésicos ultrapassam a barreira placentária, com exceção dos bloqueadores neuromusculares, sendo, infelizmente, impossível anestesiar a mãe de maneira seletiva sem deprimir o feto (BENSON \& THURMON, 1987). A permeabilidade da placenta está relacionada à sua classificação e às propriedades físico-químicas dos fármacos, sendo que a placenta endoteliocorial das cadelas facilita a passagem dos anestésicos para os fetos devido a sua espessura menor (HALL et al., 2001). Porém, mais importante que o tipo de placenta são as características físico-químicas dos fármacos, como o peso molecular, grau de ligação às proteínas plasmáticas, lipossolubilidade e grau de ionização (HALL et al., 2001).

Os anestésicos inalatórios provocam depressão fetal, a qual é diretamente proporcional à profundidade anestésica da mãe. Portanto, é importante manter a anestesia em um plano adequado para o procedimento anestésico, já que níveis profundos de anestesia causam hipotensão materna, diminuição do fluxo sangüíneo uterino, com hipóxia e acidose fetal (THURMON et al., 1996).

A gestação provoca diversas alterações fisiológicas que podem interferir no procedimento anestésico (PASCOE \& MOON, 2001). Ocorre aumento da freqüência cardíaca e respiratória, do débito cardíaco e do consumo de oxigênio, além da redução da capacidade residual funcional (THURMON et al., 1996; HALL et al., 2001; PASCOE \& MOON, 2001; ROBERTSON \& MOON, 2003).

OLIVA et al. (2000) encontraram discreta elevação da freqüência cardíaca e manutenção dos valores de pressão arterial com o uso do sevofluorano como agente de manutenção anestésica em cães.

KAZAMA \& IKEDA (1985) descreveram redução da pressão arterial média de maneira dose- dependente, com valores de 59,3 \pm 24,3; 43,9 $\pm 20,6$; $30,2 \pm 21,2 \mathrm{mmHg}$ para 1,$0 ; 1,5$ e 2,0 CAM de sevofluorano, respectivamente. Em cães anestesiados com sevofluorano, a ventilação manteve-se inalterada nas concentrações de 1,0 e 1,5 CAM, apresentando maior depressão a 2,0 CAM (MUTOH et al., 1997). Os valores da freqüência respiratória apresentaram redução somente na concentração mais elevada, permanecendo o volume corrente constante durante todos os estágios da anestesia. Os valores da $\mathrm{PaCO}_{2}$ elevaram-se somente quando se utilizou 2 CAM, ocorrendo redução concomitante do pH sangüíneo (MUTOH et al., 1997).

Considerando-se que a literatura disponível limita-se a investigar os efeitos dos procedimentos anestésicos durante o trabalho de parto e/ou da operação cesariana, mas não ao longo da gestação, objetivou-se avaliar como a anestesia geral inalatória, com o sevofluorano, interfere nos parâmetros fisiológicos de cadelas gestantes.

\section{MATERIAL E MÉTODOS}

Este estudo foi aprovado pela Comissão de Ética e Biossegurança na Experimentação Animal do Curso de Medicina Veterinária da UNESP-Câmpus de Araçatuba com o protocolo de número 45/02.

Foram utilizadas nove cadelas sem raça definida, sadias, com peso médio de $15,86 \mathrm{~kg} \pm 2,7$ e idade de $3 \pm 1,6$ anos. Foram submetidas a exames físicos e laboratoriais, como hemograma e coproparasitológico, antes do início do experimento a fim de se detectar alterações que pudessem interferir no procedimento anestésico, sendo o tratamento instituído se houvesse alguma alteração.

As cadelas foram acompanhadas quanto ao estado reprodutivo para a determinação do pró-estro, que foi definido através da visualização dos sinais clínicos como sangramento vaginal e edema vulvar, sendo então realizadas as citologias vaginais para a determinação do estro. A cobertura das cadelas foi realizada por monta natural ou inseminação artificial, esta última adotada caso a cadela não aceitasse o macho. O diagnóstico de gestação foi realizado no $30^{\circ}$ dia após a última cobertura, através do exame ultrasonográfico de acordo com KEALY \& McALLISTER (2000).

O momento de submeter a cadela ao procedimento anestésico (45 dias de gestação) foi definido conforme técnica descrita por FELDMAN \& NELSON (1996), pela qual se preconiza a realização de radiografia abdominal da mãe. Por meio dessa radiografia é possível realizar a contagem do número de fetos existentes, uma vez que o crânio e a coluna se 
tornam radiopacos entre 44 e 46 dias de gestação e também através da determinação do primeiro dia de diestro. As cadelas não gestantes foram anestesiadas aleatoriamente quando a citologia vaginal era característica de anestro.

Utilizaram-se então dois grupos de acordo com o estado reprodutivo em que os animais se encontravam: G1 na condição não gestante e G2 aos 45 dias de gestação, perfazendo nove cadelas que participaram de ambos os grupos, atuando, desta maneira, como controle delas mesmas.

Foi realizado jejum sólido de doze horas e hídrico de duas horas antes de cada procedimento anestésico. A tricotomia da região da veia cefálica foi realizada para a colocação de cateteres para a aplicação do agente indutor e para a fluidoterapia, e da região da artéria femoral para a colheita de sangue arterial.

Antes da aplicação da medicação préanestésica (M0) e após 15 minutos (M1), foram avaliados os seguintes parâmetros: temperatura retal (TR) através do termômetro clínico, freqüências cardíaca e respiratória (FC e f) através de estetoscópio, pressão arterial obtida pelo método não-invasivo ${ }^{\mathrm{a}} \mathrm{e}$ tempo de perfusão capilar (TPC) através da compressão digital da mucosa oral. A pressão arterial obtida pelo método não-invasivo foi avaliada com o manguito posicionado na base da cauda do animal, obtendo-se desta forma a pressão arterial sistólica, média e diastólica (PAS, PAM e PAD). Foram realizadas três aferições consecutivas de cada pressão, sendo o valor obtido de cada tipo de pressão submetido à média aritmética para a obtenção do valor final de cada uma. Em seguida, foi colhida uma amostra de $1 \mathrm{ml}$ de sangue da artéria femoral para realização de análise hemogasométricab.

Os animais foram tranqüilizados com acepromazina $^{c}$ na dose de $0,05 \mathrm{mg} \mathrm{kg}^{-1}$ pela via intravenosa (IV) e, 15 minutos após, foi aplicado o propofol ${ }^{\mathrm{d}}$ na dose de $5 \mathrm{mg} \mathrm{kg}^{-1}$ também IV. Após a perda total dos reflexos protetores, as cadelas foram posicionadas em decúbito dorsal e realizou-se a intubação orotraqueal, iniciando-se a fase de manutenção da anestesia com a conexão ao sistema circular de anestesia ${ }^{e}$ e à vaporização do sevofluorano ${ }^{\mathrm{f}}$ através de vaporizador calibradog diluído em oxigênio a 100\%. Foi utilizado um fluxo de 1 a 1,5 $\mathrm{lmin}^{-1}$ em média, na concentração necessária para manter o plano II do III estágio anestésico, segundo a classificação de Guedel, mantendo-se a anestesia por 60 minutos. A fluidoterapia foi realizada com Ringer, com lactato na velocidade de $10 \mathrm{ml} \mathrm{kg}^{-1} \mathrm{~h}^{-1}$, em todas as cadelas após a indução anestésica.
Após 15 minutos da estabilização da anestesia (M2), os parâmetros foram avaliados a cada 15 minutos, totalizando 60 minutos (M3, M4, M5 e M6). Após o início da vaporização do anestésico, foi inserido um cateter 20G na artéria femoral para a colheita de sangue para a realização da hemogasometria, no início da manutenção anestésica (M2) e aos 60 minutos de manutenção (M6).

Durante a manutenção da anestesia, foram avaliados adicionalmente a saturação de oxigênio na hemoglobina ${ }^{\text {h }}$ e a pressão parcial de dióxido de carbono no ar expiradoi. A recuperação anestésica foi acompanhada através da aferição dos tempos necessários para a extubação e início da deambulação.

A análise estatística foi realizada através da Análise de Variância nas variáveis com distribuição normal, sendo as médias comparadas através do teste de Tukey (ZAR, 1999). As variáveis que não apresentaram distribuição normal foram analisadas utilizando o teste de Wilcoxon para comparar os grupos em cada momento e o teste de Friedman para comparar os momentos em cada grupo, seguido do teste de Dunn para comparações múltiplas. As análises estatísticas foram consideradas significativas quando $\mathrm{P}<0,05$. Os resultados foram expressos em média \pm erro padrão da média.

\section{RESULTADOS E DISCUSSÃO}

A FC nas cadelas gestantes, antes da anestesia, apresentou valores superiores aos observados na mesma cadela não gestante, como observado por SKERMAN et al. (1991), que afirmaram ocorrer aumento na gestação, sendo essa elevação necessária para suprir a demanda de oxigênio e nutrientes para a mãe e para os fetos (HALL et al., 2001).

Durante a manutenção anestésica, contudo, as cadelas não gestantes mantiveram valores de FC bastante estáveis e sem diferença significativa em relação aos momentos basais. Nas gestantes, apesar de haver redução em alguns momentos (M3 e M4), em relação aos basais, considera-se que biologicamente a variação desse parâmetro foi pequena e perfeitamente aceitável para um animal submetido à anestesia inalatória (Tabela 1).

Neste trabalho, não se verificaram elevações da FC durante a anestesia com sevofluorano, como observado por OLIVA et al. (2000), em nenhum dos grupos avaliados, provavelmente pela discreta redução da PA (Tabela 1), devido à baixa concentração de anestésico inalatório utilizada. 
Tabela 1 - Variáveis cardiovasculares e respiratórias de cadelas não gestantes (G1) e gestantes (G2) pré-tratadas com acepromazina e propofol e anestesiadas com sevofluorano.

\begin{tabular}{|c|c|c|c|c|c|c|c|c|}
\hline Variáveis & & M0 & M1 & M2 & M3 & M4 & M5 & M6 \\
\hline \multirow[t]{2}{*}{ FC (bpm) } & G1 & $97 \pm 5 \mathrm{aB}$ & $89 \pm 7 a B$ & $90 \pm 5 a$ & $105 \pm 7 a$ & $103 \pm 6 a$ & $103 \pm 7 a$ & $103 \pm 5 a$ \\
\hline & G2 & $115 \pm 5 \mathrm{abA}$ & $126 \pm 6$ aA & $112 \pm 8 a b$ & $107 \pm 5 b$ & $108 \pm 6 b$ & $113 \pm 5 a b$ & $115 \pm 6 a b$ \\
\hline \multirow[t]{2}{*}{$\mathrm{f}(\mathrm{mpm})$} & G1 & $58 \pm 12 a$ & $32 \pm 6 a b$ & $15 \pm 2 b$ & $14 \pm 2 b$ & $14 \pm 2 b$ & $17 \pm 2 b$ & $15 \pm 1 b$ \\
\hline & G2 & $65 \pm 12 a$ & $39 \pm 8 b$ & $21 \pm 3 b$ & $20 \pm 4 b$ & $20 \pm 4 b$ & $22 \pm 4 b$ & $22 \pm 4 b$ \\
\hline \multirow[t]{2}{*}{ PAS (mmHg) } & G1 & $132 \pm 2 \mathrm{a}$ & $115 \pm 5 a b A$ & $98 \pm 4 \mathrm{bA}$ & $95 \pm 5 b A$ & $96 \pm 5 b$ & $108 \pm 5 b$ & $106 \pm 7 b$ \\
\hline & G2 & $122 \pm 4 a$ & $96 \pm 4 \mathrm{bcB}$ & $84 \pm 3 \mathrm{~dB}$ & $85 \pm 3 c d B$ & $87 \pm 3 b c d$ & $92 \pm$ 3bcd & $98 \pm 4 b$ \\
\hline \multirow[t]{2}{*}{ PAM (mmHg) } & G1 & $92 \pm 4 a$ & $72 \pm 3 b$ & $64 \pm 3 b$ & $64 \pm 4 b$ & $67 \pm 5 b$ & $77 \pm 4 \mathrm{abA}$ & $76 \pm 6 a b$ \\
\hline & G2 & $83 \pm 5 a$ & $65 \pm 3 b c$ & $56 \pm 3 c$ & $57 \pm 2 b c$ & $56 \pm 3 b c$ & $60 \pm 2 b c B$ & $66 \pm 3 b$ \\
\hline \multirow[t]{2}{*}{ PAD (mmHg) } & G1 & $65 \pm 5 \mathrm{aA}$ & $52 \pm 4 a b$ & $42 \pm 4 b$ & $43 \pm 4 b$ & $47 \pm 5 b$ & $53 \pm 4 a b$ & $56 \pm 6 a b$ \\
\hline & G2 & $54 \pm 5 a B$ & $43 \pm 5 b$ & $37 \pm 3 b$ & $37 \pm 2 b$ & $36 \pm 2 b$ & $39 \pm 2 b$ & $44 \pm 3 b$ \\
\hline \multirow[t]{2}{*}{$\mathrm{TR}\left({ }^{\circ} \mathrm{C}\right)$} & G1 & $38,8 \pm 0,16 \mathrm{aA}$ & $38,6 \pm 0,1 \mathrm{aA}$ & $37,8 \pm 0,2 b$ & $37,7 \pm 0,2 \mathrm{bc}$ & $37,4 \pm 0,2 \mathrm{bcd}$ & $37,3 \pm 0,2 \mathrm{~cd}$ & $37,2 \pm 0,3 d$ \\
\hline & G2 & $38,3 \pm 0,1 \mathrm{aB}$ & $38 \pm 0,1 \mathrm{aB}$ & $37,4 \pm 0,2 b$ & $37,2 \pm 0,2 b c$ & $37,1 \pm 0,2 \mathrm{bc}$ & $37 \pm 0,2 c$ & $37 \pm 0,2 c$ \\
\hline \multirow[t]{2}{*}{$\mathrm{ETCO}_{2}(\mathrm{mmHg})$} & G1 & $*$ & $*$ & $28,8 \pm 2,7$ & $27,7 \pm 1,8$ & $27,4 \pm 2$ & $26,3 \pm 2,3$ & $26,2 \pm 2,1$ \\
\hline & G2 & $*$ & $*$ & $28,6 \pm 1,5$ & $27,8 \pm 1,7$ & $27,6 \pm 1,2$ & $25,2 \pm 2,3$ & $26 \pm 2,1$ \\
\hline \multirow[t]{2}{*}{$\mathrm{SpO}_{2}(\%)$} & G1 & $*$ & $*$ & $97 \pm 0,3$ & $97 \pm 0,5$ & $97 \pm 0,4$ & $97 \pm 0,3$ & $97 \pm 0,4$ \\
\hline & G2 & $*$ & $*$ & $97 \pm 0,4$ & $97 \pm 0,5$ & $97 \pm 0,5$ & $97 \pm 0,4$ & $97 \pm 0,5$ \\
\hline Concentração anestésica & G1 & $*$ & $*$ & $3,06 \pm 0,3$ & $3,04 \pm 0,3$ & $2,91 \pm 0,3$ & $2,64 \pm 0,1$ & $2,66 \pm 0,2$ \\
\hline de sevofluorano & G2 & $*$ & $*$ & $2,72 \pm 0,1$ & $2,6 \pm 0,1$ & $2,61 \pm 0,1$ & $2,5 \pm 0,07$ & $2,5 \pm 0,07$ \\
\hline
\end{tabular}

Valores expressos em média \pm erro padrão da média.

* = variável não mensurada

FC = freqüência cardíaca, $\mathrm{f}=$ freqüência respiratória, $\mathrm{PAS}$ = pressão arterial sistólica, $\mathrm{PAM}=$ pressão arterial média, $\mathrm{PAD}=$ pressão arterial diastólica, $\mathrm{TR}=$ temperatura retal, $\mathrm{ETCO}_{2}=$ pressão parcial de dióxido de carbono no ar expirado, $\mathrm{SpO}_{2}=\mathrm{s}_{2}$ saturação de oxigênio na hemoglobina

Letras maiúsculas diferentes indicam diferenças significativas entre grupos.

Letras minúsculas diferentes indicam diferenças significativas entre momentos dentro do mesmo grupo.

O trabalho cardíaco na paciente gestante é maior e resulta, portanto, em reserva cardíaca menor, predispondo a descompensação em pacientes previamente estáveis (GREENE, 1995). Durante a gestação, a resposta através de mecanismos cardiovasculares compensatórios pode ser mais lenta (ROBERTSON \& MOON, 2003).

Houve redução da pressão arterial sistólica (PAS) em todos os momentos da manutenção anestésica, nos dois grupos avaliados (Tabela 1). No G2, os valores foram inferiores aos obtidos em G1, em M1, M2 e M3, corroborando dados de PROBST \& WEBB (1983), que observaram diminuição da PAS em cadelas próximas ao momento do parto, porém anestesiadas com halotano e induzidas com tiamilal sódico, sem a utilização da MPA.

A PAM reduziu-se após a MPA em todos os animais (Tabela 1), com valores inferiores ao normal em alguns momentos da manutenção no G1 e em todos os momentos no G2. A PAD apresentou comportamento idêntico ao da PAM, mas apresentou valores inferiores nas gestantes apenas em M0.
Essa redução é decorrente das alterações fisiológicas da gestação (BROOKS \& KEIL, 1994), dos efeitos hipotensores da acepromazina pelo bloqueio dos receptores $\alpha$ adrenérgicos e da depressão dos centros motores centrais promovida pelos fenotiazínicos (FARVER et al., 1986; BROCK, 1994).É também decorrente do propofol, que promove diminuição da PAS, PAM e PAD, sem aumento compensatório da FC (SHORT \& BUFALARI, 1999) e do sevofluorano, que promove redução da PA dose dependente, devido à diminuição da resistência vascular periférica em concentrações de 1,2 CAM (BRAZ \& CASTIGLIA, 2000).

Ao correlacionar-se as variações de FC e PA de ambos os grupos (Tabela 1), verificam-se que as respostas de aumento da FC esperadas nos momentos de valores mais baixos de PA não ocorreram nas gestantes, o que confirma afirmações de GREENE (1995) e ROBERTSON \& MOON (2003). Pode-se sugerir, portanto, que deva haver uma atenção maior na manutenção da PA em valores adequados (PAM acima de $70 \mathrm{mmHg}$ ) nas pacientes gestantes, uma vez que sua 
resposta compensatória possa estar reduzida. Além do que, o fluxo sangüíneo fetal não é auto-regulável, e depende diretamente dos valores da pressão arterial (ROBERTSON \& MOON, 2003).

Após a MPA(M1), nos dois grupos ocorreu diminuição da freqüência respiratória (f) o que está de acordo com CORTOPASSI \& FANTONI (2002), que descreveram a redução dessa variável e do volume minuto $\left(\mathrm{V}_{\mathrm{M}}\right)$ após a aplicação de um fenotiazínico. $\mathrm{O}$ propofol também promove diminuição da $\mathrm{f}$ dose dependente, com redução do volume corrente (Vt) (BRAZ \& CASTIGLIA, 2000).

Ocorreu elevação da $\mathrm{PaO}_{2}$ e da $\mathrm{SatO}_{2}$ (tabela 2) no sangue arterial, nos dois grupos, a partir de M2, devido ao fornecimento de $\mathrm{O}_{2}$ a $100 \%$, garantindo a oxigenação sangüínea durante o procedimento anestésico. No entanto, os valores da $\mathrm{SpO}_{2}$ obtidos pelo oxímetro de pulso foram sempre inferiores aos obtidos pela análise hemogasométrica, porém dentro dos valores considerados normais para a espécie, sem risco de hipóxia para os animais (MOYLE et al., 1994).É importante considerar que a $\mathrm{SpO}_{2}$ sofre interferência pela baixa perfusão tecidual que muitas vezes ocorre na anestesia (NUNES, 2002).

Houve redução dos valores de $\mathrm{pH}$ nos dois grupos ao longo do procedimento anestésico; porém, somente no grupo G1 essa diminuição foi significativa em M2 e M6 quando comparada a M0. Essa redução pode ser devida à diminuição da freqüência respiratória, porém não ocorreram elevações significativas da $\mathrm{PaCO}_{2}$ e nos valores de bicarbonato (Tabela 2).
Em relação ao déficit de bases, que avalia o componente metabólico, observou-se diminuição em G1 e G2, embora sem diferença significativa, segundo valores de ROBERTSON (1989), que considera normal os valores de $0 \pm 4 \mathrm{mmol} \mathrm{l}^{-1}$. Esta diminuição poderia indicar a ocorrência de acidose metabólica; porém, isso não pode ser totalmente afirmado, pois os valores de bicarbonato não se alteraram, encontrando-se dentro dos limites normais para a espécie.

Durante a manutenção anestésica, os valores de $\mathrm{f}$ foram inferiores aos basais nos dois grupos. Contudo, esta diminuição foi provavelmente compensada pelo aumento do volume corrente, mantendo-se assim o volume minuto. Isso caracteriza a ausência de depressão respiratória, já que os valores de pH sangüíneo, PaCO e ETCO ${ }_{2}$ estiveram em valores considerados aceitáveis e sem alteração significativa ao longo da anestesia. Esses dados estão de acordo com MUTOH et al. (1997), que verificaram elevação da $\mathrm{PaCO}_{2}$ e conseqüente redução do $\mathrm{pH}$ somente quando utilizaram 2 CAM de sevofluorano na espécie canina.

Não foi observado aumento da $\mathrm{f}$ nas gestantes, como relataram HALL et al. (2001) e PROBST \& WEBB (1983), devido ao tempo de gestação do experimento ser de 45 dias e não de 60 dias, quando as alterações mecânicas são mais evidentes, culminando em maiores elevações dessa variável.

A diminuição da TR (Tabela 1) nos dois grupos deve-se à utilização da acepromazina, do propofol e do sevofluorano, os quais possuem ação depressora sobre os mecanismos de termorregulação no hipotálamo, além de produzirem vasodilatação

Tabela 2 - Variáveis hemogasométricas de cadelas não gestantes (G1) e gestantes (G2) pré-tratadas com acepromazina e propofol e anestesiadas com sevofluorano.

\begin{tabular}{|c|c|c|c|c|}
\hline Variáveis & & M0 & M2 & M6 \\
\hline \multirow[t]{2}{*}{$\mathrm{pH}$} & G1 & $7,4 \pm 0,01 a$ & $7,36 \pm 0,01 b$ & $7,37 \pm 0,01 b$ \\
\hline & G2 & $7,39 \pm 0,03 a$ & $7,30 \pm 0,03 a$ & $7,30 \pm 0,06 a$ \\
\hline \multirow[t]{2}{*}{$\mathrm{PaCO}_{2}(\mathrm{mmHg})$} & G1 & $30,11 \pm 2,68$ & $32,64 \pm 3$ & $33,08 \pm 2,53$ \\
\hline & G2 & $32 \pm 2,74$ & $40,48 \pm 4,61$ & $43,86 \pm 13,25$ \\
\hline \multirow[t]{2}{*}{$\mathrm{PaO}_{2}(\mathrm{mmHg})$} & G1 & $103,6 \pm 3,7 b$ & $342,1 \pm 25,5 a$ & $349,8 \pm 21,4 a$ \\
\hline & G2 & $103 \pm 5,5 b$ & $332,5 \pm 13,2 \mathrm{a}$ & $298 \pm 37,2 \mathrm{a}$ \\
\hline \multirow[t]{2}{*}{$\mathrm{HCO3}^{-}(\mathrm{mmol} / \mathrm{l})$} & G1 & $18,7 \pm 1,4$ & $18 \pm 1,2$ & $18,9 \pm 1,2$ \\
\hline & G2 & $19,1 \pm 1,5$ & $19,6 \pm 1,2$ & $18,7 \pm 2,2$ \\
\hline \multirow[t]{2}{*}{$\mathrm{BE}(\mathrm{mmol} / \mathrm{l})$} & G1 & $-4,4 \pm 1,0 \mathrm{a}$ & $-5,9 \pm 0,8 a$ & $-4,9 \pm 0,9 a$ \\
\hline & G2 & $-4,3 \pm 1,6 a$ & $-5,9 \pm 1,0 a$ & $-6,7 \pm 1,5 a$ \\
\hline \multirow[t]{2}{*}{$\mathrm{SatO}_{2}(\%)$} & G1 & $97,7 \pm 0,2 b$ & $99,6 \pm 0,1 \mathrm{a}$ & $99,7 \pm 0,04 a$ \\
\hline & G2 & $97,0 \pm 0,7 b$ & $99,7 \pm 0,03 a$ & $99,5 \pm 0,1 \mathrm{a}$ \\
\hline
\end{tabular}

Valores expressos em média \pm erro padrão da média.

$\mathrm{pH}=$ potencial hidrogeniônico $\mathrm{PaCO}_{2}=$ pressão parcial arterial de dióxido de carbono $\mathrm{PaO}_{2}=$ pressão parcial arterial de oxigênio $\mathrm{HCO}=$ bicarbonato $\mathrm{BE}=$ déficit de bases $\mathrm{SatO}_{2}=$ saturação de oxigênio na hemoglobina

Letras minúsculas diferentes indicam diferenças significativas entre momentos dentro do mesmo grupo.

Ciência Rural, v.36, n.3, mai-jun, 2006. 
periférica e relaxamento muscular (MASSONE, 2003; SPINOSA \& GÓRNIAK, 2002).

A concentração de anestésico inalatório requerido para as cadelas gestantes foi o mesmo para o grupo não-gestante, entre 2,5 e 3\% de sevofluorano, durante todo o procedimento anestésico, discordando de estudos prévios realizados por PALAHNIUK et al. (1974), os quais relataram diminuição do requerimento anestésico do halotano, isofluorano e metoxifluorano em ovelhas no terço-final de gestação. Essa redução seria explicada pelos efeitos sedativos da progesterona e das endorfinas que se encontram elevadas na gestação. Porém, no atual experimento, o tempo de gestação foi de aproximadamente 45 dias, ou seja, no início do terço final de gestação, enquanto que as ovelhas utilizadas naquele estudo encontravam-se no final da gestação.

O sevofluorano, por possuir baixo coeficiente de solubilidade sangüínea, contribuiu para uma recuperação rápida e tranqüila da anestesia, com valores médios para a extubação de 8,8 e 6,6 minutos e para o início da deambulação de 26,3 e 17,1 minutos para o grupo G1 e G2, respectivamente.

\section{CONCLUSÃO}

Nas condições experimentais utilizadas, é possível concluir que a anestesia geral inalatória com o sevofluorano, em animais pré-tratados com acepromazina e propofol, não interfere de forma importante nas variáveis cardiorrespiratórias na cadela em gestação de 45 dias, podendo ser utilizado com segurança para as mães, no caso de necessidade de intervenção cirúrgica e/ou anestésica durante esse período.

\section{FONTES DE AQUISIÇÃO}

a Dixtal - DX 2710

b Drace - AGS 12

c Acepran 0,2\% - Univet S.A. Industria Veterinária

d Propovan - Cristália Produtos Químicos e Farmacêuticos Ltda

e Oxigel - mod. 800

f Sevocris - Cristália Produtos Químicos Farmacêuticos Ltda

g Takaoka - mod. 1225

h Takaoka - mod. 9505

${ }^{i}$ Dixtal, Criticare Systems Inc. Poet. TE

\section{AGRADECIMENTOS}

À Fundação de Amparo à Pesquisa do Estado de São Paulo (FAPESP), pelo apoio financeiro.

\section{REFERÊNCIAS}

BENSON, G.J.; THURMON, J.C. Special anesthetic considerations for caesarean section. In: SHORT C.E. Principles and practice of veterinary anesthesia. Baltimore: Williams \& Wilkins, 1987. Cap.13, p.337348 .

BRAZ, R.C.; CASTIGLIA, Y.M.M. Temas de anestesiologia para o curso de graduação em medicina. 2.ed. São Paulo: Artes Médicas, 2000. p.315.

BROCK, N. Acepromazine revised. Can Vet J, v.35, p.458459, 1994

BROOKS, V.L.; KEIL, L.C. Hemorrhage decreases arterial pressure sooner in pregnant compared with nonpregnant dogs: role of baroreflex. Am J Physiol, v.266, pt.2, p.H1610H1619, 1994.

CORTOPASSI, S.R.G.; FANTONI, D.T. Medicação préanestésica. In: FANTONI, D.T.; CORTOPASSI, S.R.G. Anestesia em cães e gatos. São Paulo: Rocca, 2002. Cap.13, p.151-158.

FARVER, T.B. et al. Cardiopulmonary effects of acepromazine and of the subsequent administration of ketamine in the dog. Am J Vet Res, v.47, p.631-635, 1986.

FELDMAN, E.C.; NELSON, R.W. Breeding, pregnancy and parturition. In: Canine and feline endocrinology and reproduction. 2.ed. Philadelphia: Saunders, 1996. Cap.18, p.559.

GREENE, S.A. Anesthetic considerations for surgery of the reproductive system. Seminars in Veterinary Medicine Surgical (Small Animal), v.10, n.1, p.2-7, 1995.

HALL, L.W. et al. Anaesthesia for obstetrics. In: Veterinary anaesthesia. 10.ed. London: Saunders, 2001. Cap.18, p.481-491.

KAZAMA, T.; IKEDA, K. The comparative cardiovascular effects and induction time of sevoflurane with isoflurane and halothane in dog. Anesthesiology, v.63, p.A17, 1985.

KEALY, J.K.; McALLISTER, H. Diagnostic radiology and ultrasonography of the dog and cat. 3.ed. Philadelphia: Saunders, 2000. p.136-173.

MASSONE, F. Anestesiologia veterinária: farmacologia e técnicas. 4.ed. Rio de Janeiro: Guanabara Koogan, 2003. 326p.

MOYLE, J.T. et al. Pulse-oximeter: principles and practice series. London: BMJ, 1994. p.134.

MUTOH, T. et al. Cardiopulmonary effects of sevoflurane compared with halothane, enflurane and isoflurane in dogs. Am J Vet Res, v.58, n.8, p.885-890, 1997.

NUNES, N. Monitoração da anestesia. In: FANTONI, D.T.; CORTOPASSI, S.R.G. Anestesia em cães e gatos. São Paulo: Roca, 2002. Cap.6, p.64-81. 
PALAHNIUK, R.J. et al. Pregnancy decreases the requirement for inhaled anesthetic agents. Anesthesiology, v.41, p.8283, 1974.

PASCOE, P.J.; MOON, P.F. Periparturient and neonatal anesthesia. Vet Clin North Am Small Anim Pract, v.31, n.2, p.315-341, 2001.

OLIVA, V.N.L.S. et al. Avaliação cardiocirculatória do sevofluorano como agente de manutenção anestésica em cães, em diferentes concentrações de oxigênio e óxido nitroso. Arq Bras Med Vet Zootec, v.52, p.130-137, 2000.

PROBST, C.W.; WEBB, A.I. Postural influence on systemic blood pressure, gas exchange, and acid/base status in the termpregnant bitch during general anesthesia. Am J Vet Res, v.44, n.10, p.1963-1965, 1983.

ROBERTSON, S.A. Simple acid base disorders. Vet Clin North Am Small Anim Pract, v.19, p.289-306, 1989.

ROBERTSON, S.A.; MOON, P.F. Anesthetic management for cesarean section in bitches. Veterinary Medicine, v.98, n.8, p.675-696, 2003.
SCHNIDER, S.M.; LEVINSON, G. Anesthesia for cesarean section. In: SCHNIDER, S.M., LEVINSON, G. Anesthesia for Obstetrics. 2.ed. Baltimore: Williams \& Wilkins, 1987. Cap.12, p.159-178.

SHORT, C.E.; BUFALARI, A. Propofol anesthesia. Vet Clin North Am Small Anim Pract, v.29, p.747-778, 1999.

SKERMAN, J.H. et al. Effects of anaesthesia on maternal/fetal circulation. Seminars Anesth, v.10, n.4, p.235-246, 1991.

SPINOSA, H.L.; GÓRNIAK, S.L. Tranqüilizantes e relaxantes musculares de ação central. In: SPINOSA, H.L. et al. Farmacologia aplicada à medicina veterinária. 3.ed. Rio de Janeiro: Guanabara Koogan, 2002. Cap.14, p.146-157.

THURMON, J.C. et al. Anesthesia for special patients: cesarean section patients. In: LUMB, W.V.; JONES, E.W. Veterinary anesthesia. 3.ed. Baltimore: Williams \& Wilkins, 1996. Cap.24, p.818-828.

ZAR, J.H. Biostatistical analysis. 4.ed. New Jersey: Prentice Hall, 1999. p.875. 